\title{
Path integral Monte Carlo study of quantum-hard sphere solids
}

Luis M. Sesé

Departamento de Ciencias y Técnicas Fisicoquímicas, Facultad de Ciencias, Universidad

Nacional de Educación a Distancia, Paseo Senda del Rey 9, 28040 Madrid, Spain

A path integral study of the $f c c, h c p$, and $b c c$ quantum hard-sphere solids is presented. Ranges of densities within the interval of reduced de Broglie wavelengths $0.2 \leq \lambda_{B}^{*} \leq 0.8$ have been analyzed using Monte Carlo simulations with Cao-Berne propagator. Energies, pressures, and structural quantities (pair radial correlation functions, centroid structure factors, and Steinhardt order parameters) have been computed. Also, applications of the Einstein crystal technique (J. Chem. Phys. 126, 164508 (2007)) have been made to compute the free energies of the $f c c$ and hcp solids. Some technical points related to the latter technique are discussed, and it is shown that these calculations produce consistent results with increasing sample sizes. The fluid-solid ( $f c c$ and $h c p$ ) equilibria have been studied, thus completing prior work by this author on the fluid- $f c c$ equilibrium. Within the accuracy attained no significant differences between the relative stabilities of the $f c c$ and $h c p$ lattices have been detected. The $b c c$ case stands apart from the other two lattices, as the simulations lead either to irregular lattices (two types) that keep some traces of bcc-memory, or to spontaneous transitions to $h c p$-like lattices. The latter transitions make manifestly clear the potential repercussions that the quantum hard-sphere behavior can have on solid-solid equilibria at low temperatures in real systems (e.g. helium).

Published in The Journal of Chemical Physics, vol.139, 044502-1/13 (2013)

Email: msese@ccia.uned.es 


\section{INTRODUCTION}

The hard sphere model ( $\sigma=$ classical collision parameter) appears as a good choice to carry out computer simulations of fluid or solid phases. Its usefulness in the classical domain is well-established. ${ }^{1-10}$ On the other hand, quantum statistical studies with this singular model (QHS) are far more involved, because of the non-analytical potential and the wave/spin nature of particles. In this regard, a number of quantum approaches have been put forward to deal with QHS $^{11-27}$ and, among them, Feynman's path integrals (PI) stand out as a most powerful tool. ${ }^{15,17-}$ ${ }^{27}$ In particular, the use of PI and/or QHS has led to an understanding of a number of general features of helium in the condensed phases, ${ }^{14-15}$ thus serving as a complement to studies with continuous potentials of this important system. ${ }^{28,29}$

Focusing attention on the modeling of solid phases, there is a good deal of results obtained with classical hard-spheres. One may mention the relative stability of the lattices facecentered cubic $(f c c)$ and hexagonal close-packed $(h c p)$, which turns out to be slightly greater for $f c c,{ }^{3,7}$ and also the fluid-solid equilibrium of colloids. ${ }^{9,10}$ However, to the knowledge of this author, only a few PI studies of the QHS face-centered cubic and body-centered cubic (bcc) lattices are available, ${ }^{15,30-32}$ and there are some reasons that indicate that further work on the QHS solids would be worthwhile to complete the picture of solids at low temperatures. For example, the $b c c$ lattice is known to play a significant role in the initial nucleation of metastable fluids, ${ }^{7,33}$ and one finds $b c c$ lattices for a substantial number of the metals on the left-hand side of the Periodic Table. ${ }^{4,33,34}$ Moreover, $b c c$ also appears under certain conditions as the structure of solid phases composed of light atoms as the helium isotopes. ${ }^{35-39}$ In this connection, the $b c c$ lattice of He isotopes is being subject of active research, ${ }^{38,41}$ and so is the $h c p-b c c$ equilibrium in ${ }^{4} \mathrm{He}$ in its own right. ${ }^{38-39}$ Furthermore, based on previous experience ${ }^{9,10,14,15,25}$ it seems clear that work on the QHS system could give more insights into the study of highly complex real systems. In this regard, it is hoped that QHS, bare or with attractive forces, may prove its usefulness for the 
understanding of hard-sphere like systems, such as colloids or ultra-hard materials, at very low temperatures.

Over the past years work by this author has dealt with a wide range of issues of the QHS fluid and solid phases. ${ }^{26-27,30-32,42-49}$ Some of them are of interest to this work: the accurate equation of state for the fluid phase reported recently, ${ }^{49}$ a preliminary study of the fluid-solid $f c c$ transition for intermediate densities, ${ }^{31,32}$ and also the computation of a number of properties of the $f c c$ and $b c c$ lattices. ${ }^{30}$ With the better computational means of today this article is devoted to extending and completing the foregoing QHS results by studying the properties of the $f c c, h c p$, and $b c c$ solid phases.

To undertake this project, a significant number of density conditions for each type of lattice, within the range of isotherms $0.2 \leq \lambda_{B}^{*} \leq 0.8 \quad\left[\lambda_{B}^{*}=\lambda_{B} / \sigma=h /\left(2 \pi m k_{B} T \sigma^{2}\right)^{1 / 2}\right]$, are analyzed. Path-integral Monte Carlo (PIMC) simulations utilizing Cao-Berne propagator ${ }^{23}$ are carried out to obtain the mechanical and structural properties. Moreover, application of the Einstein crystal technique (EC) for QHS, as put forward in Ref. 31, is made to obtain the $f c c$ and the $h c p$ free energies. In this connection, some further aspects of the EC implementation are discussed in the present work. By so doing, and with the use of the recent QHS-fluid equation of state, ${ }^{49}$ a more accurate examination of the QHS melting-freezing transition is achieved in this work. Furthermore, a detailed investigation of the mechanical and structural properties of the peculiar solids (bcc-q) arising from the PIMC $b c c$ simulations is conducted. Free energies for the $b c c$-q cases are not calculated, because of the uncertainties brought about by their symmetry features. In spite of this, clear evidence of the principal role played by the QHS behavior in the $b c c \rightarrow h c p$ transition at low temperatures is obtained.

The outline of this article is as follows. Section II describes the basic theory, Sec. III the computational details, and Sec. IV the results and discussion. Finally, Sec. V collates the main conclusions of this work. 


\section{THEORY}

A canonical ensemble $(N, V, T)$ of identical hard spheres with diameter $\sigma$ and mass $m$ will be used to describe the QHS solid system. The bulk number density will be denoted by $\rho_{N}=N / V$. For convenience, this quantity will be expressed throughout this article in terms of the reduced density $\rho_{N}^{*}=\rho_{N} \sigma^{3}$. Note that in the canonical simulation of a given state point the bulk number density is kept fixed, i.e. $\rho_{N}=N_{S} / V_{S}$, where $N_{S}$ and $V_{S}$ are the number of particles and the volume of the central box, respectively. This intensive quantity is central to the definition of the thermodynamic limit (i.e. $T-\lim : N_{S}, V_{S} \rightarrow \infty, \quad \rho_{N}=N / V=N_{S} / V_{S}=$ finite constant $>0$ ), in which simulation work is deeply rooted. Hereafter, angular brackets $\langle\ldots\rangle$ will stand for an ensemble average, and quantum exchange will be neglected.

\section{A. PI concepts}

Every particle of the QHS system is described by an elastic necklace composed of $P$ beads numbered $t=1,2, \ldots, P$. Theoretical Trotter accuracy $(P \rightarrow \infty)^{50}$ is thus replaced by statistical convergence, which will be assumed to occur for a finite $P$ hereafter. The general form of the PI canonical partition function is given by $\left(\beta=1 / k_{B} T\right)$

$$
Z_{P I}=C\left(\frac{m P}{2 \pi \beta \hbar^{2}}\right)^{3 N P / 2} \int \prod_{i=1}^{N} \prod_{t=1}^{P} d \mathbf{r}_{i}^{t} \times \exp \left[-\beta W_{N P}\right]
$$

where $C=1$ for solids, $C=(N !)^{-1}$ for fluids, $\mathbf{r}_{i}^{t}$ denotes the spatial coordinates of bead $t$ (i.e. imaginary time $\beta \hbar t / P$ ) belonging to necklace $i$, and $W_{N P}$ is the effective potential ruling the model system composed of $N \times P$ beads. To deal efficiently with the special features of the QHS interactions in the vicinity of the hard core, ${ }^{19} W_{N P}$ has been built with Cao-Berne pair action. ${ }^{23}$ By so doing, physically significant results can be obtained with a reduced $P$ value. ${ }^{15,26,49}$ The reader may find the related formulas elsewhere. ${ }^{30,48}$ 
For the current purposes three key quantities are the pressure $p$, the internal energy $E$, and the pair instantaneous correlation function $g_{E T 2}(r)$. The QHS pressure involves the other two quantities and reads as ${ }^{51}$

$$
p=\frac{2 E}{3 V}+\frac{\rho_{N}^{2} \pi \sigma^{3} \hbar^{2}}{3 m}\left(\frac{d^{2} g_{E T 2}(r)}{d r^{2}}\right)_{r=\sigma+}
$$

where the pair radial correlation function (instantaneous) can be cast as the "equal-time" average

$$
g_{E T 2}(r)=\left\langle P^{-1} \sum_{t=1}^{P} \delta\left(r-r_{i j}^{t}\right)\right\rangle, \quad r_{i j}^{t}=\left|\mathbf{r}_{i}^{t}-\mathbf{r}_{j}^{t}\right|
$$

and the internal energy is given by

$$
E=\frac{3 N P}{2 \beta}-\frac{m P}{2 \beta^{2} \hbar^{2}}\left\langle\sum_{i=1}^{N} \sum_{t=1}^{P}{ }^{\prime}\left(\mathbf{r}_{i}^{t+1}-\mathbf{r}_{i}^{t}\right)^{2}-\sum_{i<j} \sum_{t=1}^{P}{ }^{\prime} B\left(\frac{r_{i j}^{t} r_{i j}^{t+1} \exp \left(m P B / 2 \beta \hbar^{2}\right)}{\sigma\left(r_{i j}^{t}+r_{i j}^{t+1}-\sigma\right)}-1\right)^{-1}\right\rangle,
$$

with $B=\left(r_{i j}^{t}-\sigma\right)\left(r_{i j}^{t+1}-\sigma\right)(1+\cos \gamma)$, where $\gamma$ is the angle between $\mathbf{r}_{i j}^{t}$ and $\mathbf{r}_{i j}^{t+1}$, and the primed sums imply $P+1 \equiv 1$.

The computation of free energies can be carried out by integrating the equation of state along isotherms to obtain Helmholtz $A$

$$
\frac{A_{2}}{R T}=\frac{A_{1}}{R T}+\int_{\rho_{1}}^{\rho_{2}} \frac{p V}{R T} \frac{d \rho_{N}}{\rho_{N}}, \lambda_{B}^{*}=\text { const. }
$$

and then Gibbs $G$ via $G=A+p V$. This route requires knowledge of $A$ at some target state point $\left(\rho_{N}, \lambda_{B}^{*}\right)_{1}$. This process is straightforward for the fluid phase, ${ }^{15,42}$ whereas it becomes highly involved for solid phases as shown below.

\section{B. Einstein crystal technique (EC)}

This technique was originally proposed by Frenkel and Ladd to obtain $A$ for classical solids. ${ }^{6}$ It has evolved into producing finer approaches, ${ }^{52}$ and it can also be adapted to carry out computations of $A$ energies for quantum solids. ${ }^{15,31,52(\mathrm{e})}$ When studying QHS solid phases EC is specially valuable in that hard cores can be dealt with in a fully consistent way. The goal is to 
devise a reversible path connecting a target state point of the $N \times P$ solid with a reference state point of same symmetry and known free energy. To do so, one utilizes the following auxiliary partition function ${ }^{15,31}$

$Z_{P I}\left(\alpha_{C}^{*}\right)=\left(\frac{m P}{2 \pi \beta \hbar^{2}}\right)^{3 N P / 2} \int \prod_{i=1}^{N} \prod_{t=1}^{P} d \mathbf{r}_{i}^{t} \times \exp \left[-\beta\left(W_{N P}+\frac{\alpha_{C}^{*}}{P} \sum_{i=1}^{N} \sum_{t=1}^{P}\left(\mathbf{r}_{i}^{t}-\mathbf{r}_{0, i}\right)^{2}\right)\right]$,

where the beads belonging to necklace $i$ are harmonically linked to the $i$-site $\mathbf{r}_{0, i}$ of the solid lattice. It is worth noting that Eq. (6) can be obtained by taking a conventional $\mathrm{N}$-particle Hamiltonian, which also includes the one-particle couplings to the lattice sites, and then by applying the PI approach ${ }^{49}$ plus the superposition of Cao-Berne pair actions. ${ }^{19,23}$ For $\alpha_{C}^{*}=0$ one retrieves the actual target state point of the quantum solid, whilst the Einstein crystal is attained with a truly large $\alpha_{C}^{*}$ value which makes $W_{N P}$ negligible. However, one notes that for sufficiently large values $\alpha_{C}^{*} \simeq \alpha_{M}^{*}$, the Einstein crystal associated with $\alpha_{M}^{*}$ yields already a fine description of the theoretical Einstein limit for the target state point. Given that $W_{N P}$, though small, turns out to be nonzero when use of $\alpha_{M}^{*}$ is made, a correction to the final free energy is needed. This correction can be obtained via perturbation theory and umbrella sampling, as explained elsewhere. ${ }^{15,31}$ It is also interesting to stress that $\alpha_{M}^{*}$ increases with $P$, the dependence being somewhat close to a linear one as indicated by numerical work (see below).

From the $\alpha_{C}^{*}$-derivative of Eq. (6) one obtains the basic equation for the free energy of the target state point

$$
A\left(\alpha_{C}^{*}=0\right)=\bar{A}^{E}\left(\alpha_{M}^{*}\right)-\int_{0}^{\alpha_{M}^{*}} d \alpha^{*}\left\langle P^{-1} \sum_{i=1}^{N} \sum_{t=1}^{P}\left(\mathbf{r}_{i}^{t}-\mathbf{r}_{0, i}\right)^{2}\right\rangle_{\alpha^{*}}
$$

where $\bar{A}^{E}$ is the free energy of the $\alpha_{M}^{*}$-Einstein crystal, and the ensemble average $\langle\ldots\rangle_{\alpha^{*}}$ to be integrated has to be determined at a number of $\alpha^{*}$ values. The practical application of Eq. (7) requires a number of further steps connected to: i) the effective reaching of the Einstein crystal; 
ii) the drifting of the center-of-mass (COM) of the solid lattice in a simulation; and iii) the behavior of the resulting computational procedure with increasing sample sizes. Most of the discussion related to i) and ii) was contained in Refs. 15 and 31, although for the sake of completeness some related remarks will be made below together with item iii).

In using PIMC simulations with sample size $N_{S} \times P$, one finds that the free energy of the target state point of the actual solid is ${ }^{31}$

$$
\begin{aligned}
& \frac{A(0)}{R T}=\frac{3 P}{2} \ln \left(\frac{\lambda_{B}^{2} \alpha_{M}}{\pi P^{2}}\right)-\int_{0}^{\alpha_{M}} d \alpha\left\langle\frac{1}{N_{S} P} \sum_{i=1}^{N_{S}} \sum_{t=1}^{P}\left(\mathbf{r}_{i}^{t}-\mathbf{r}_{0, i}\right)^{2}\right\rangle_{\alpha, C O M}+ \\
& \frac{\left\langle W_{N P}\right\rangle_{\alpha_{M}}^{C O M}}{R T}-\frac{3}{2 N_{S}} \ln \left(\frac{\lambda_{B}^{2} \alpha_{M}}{\pi P}\right)+\frac{1}{N_{S}} \ln \left(\frac{\rho_{N} \lambda_{B}^{3}}{N_{S}^{3 / 2} P^{1 / 2}}\right),
\end{aligned}
$$

where $\alpha=\beta \alpha^{*}$. The first contribution will be denoted by $\left(A_{1} / R T\right)$ and is the $A$ reduced energy of the Einstein crystal computed at $\alpha_{M}$. The second contribution, $\left(A_{2} / R T\right)$, is the reversible work obtained with simulations including the constraint of fixed COM for the solid lattices. The third contribution, $\left(A_{3} / R T\right)$, is the perturbation correction alluded to above obtained under the constraint of fixed COM. The fourth and fifth contributions, $\left(A_{4} / R T\right)$ and $\left(A_{5} / R T\right)$, stand for the finite corrections brought about by the COM constraint for the Einstein and the actual crystal calculations, respectively.

It is worth noting that a reliable definition of the lattice COM in PIMC simulations is given by the global center of mass of the $N_{S} \times P$ beads in the sample. Accordingly, the fifth contribution in Eq. (8) is obtained by extending the usual Wigner-Seitz argument ${ }^{31,52(a)}$ to the $N_{S} \times P$ sample. This "classical" procedure is motivated by the fact that, all along the $\alpha_{C}^{*}-$ path, the configuration integral in Eq. (6) always represents an effective system composed of $N_{S} \times P$ particles, and never a system composed of just $N_{S}$ particles. Although trivial, one notes that by taking $W_{N P}=0$, the Einstein crystal energy is independent of $\alpha_{C}^{*} / P$ and shows $3 N_{S} P$ degrees 
of freedom (i.e. equipartition theorem). Therefore, the corrections $\left(A_{4} / R T\right)$ and $\left(A_{5} / R T\right)$ in Eq. (8) are dealt with in the same manner regarding the number of degrees of freedom of the two model systems EC and PI employed. There is another choice for $A_{5} / R T$ which is just the classical expression (i.e. $P=1$ ) and is based on the global features of the COM translation. ${ }^{52(e)}$ The differences between both choices can be made negligible in practical EC-PI applications, as shown later on.

To understand the usefulness of Eq. (8) it will suffice to concentrate on the situations of practical interest and use numerical information available. As regards the first and second contributions in Eq. (8), numerical calculations indicate that when $P$ increases the behavior of $A_{1} / R T$ is somehow compensated by that of $A_{2} / R T$, as expected on physical grounds. The role of $A_{3} / R T$ is just to smooth out the differences brought about by the different choices of $\alpha_{M}$ and $P$ that can be made. With respect to $\left(A_{4} / R T\right)$ and $\left(A_{5} / R T\right)$, one notes that by setting $P=1$ (i.e. the classical situation) both quantities tend to zero in the $T-\lim$. It is also known that in PI applications the quantum limit is reached before the $T-\lim$ is. Then, by assuming that the quantum limit to describe the particles has been reached with a finite $P$, the $T$-lim also makes these two COM-corrections tend to zero, as expected. The reader will find confirmation of all these facts in the results given in this article.

Nevertheless, some important remarks regarding $\mathrm{COM}$ calculations remain to be highlighted. First, there is evidence that the fixed-COM corrections depend on the geometry and volume of the simulation box, ${ }^{53}$ and corrections based on Ref. 52 (a) do not show such dependence This effect can affect extrapolations to the $T$-lim and, to the knowledge of this author, a general formula/procedure to deal exactly with this issue has not yet been found. Fortunately, the magnitude of this effect turns out to be quantitatively small when use of a sufficiently large $N_{S}$ is made. On the other hand, one must realize that COM corrections prove 
to be necessary ${ }^{52(a)}$ to obtain consistent results when working with finite samples (see below), and one cannot rule out their use. Second, from a practical point of view, one has to be aware that in Eq. (8) the $N_{S}$ and the $P$ effects are intertwined. Accordingly, as the quantum effects and $P$ increase, the number $N_{S}$ must also be augmented, so as to keep the corrections small and produce consistent results. Third, in general all the foregoing COM drawbacks can always be greatly reduced by using a sufficiently large $N_{S}$. In particular, for $N_{S} \gg P$ the role of $P$ in $A_{5} / R T$ would be negligible, and hence so would the difference between $A_{5} / R T$ and its (classical) counterpart correction obtained by setting $P=1$. Fourth, it is clear then the importance of using PI techniques that reduce $P$ (i.e. pair actions, ${ }^{23,28}$ higher-order propagators $)^{29}$ in undertaking these calculations. This work is within the latter strategy and its results show the full consistency of the procedure based on Eqs. (6)-(8).

\section{Order parameters}

In addition to $g_{E T 2}(r)$ a number of significant parameters related to the PI centroids (i.e. necklace centers of mass) are used in this work to characterize order in the solid phases. These parameters are described below.

a) The pair radial correlation function between centroids, which can be cast as

$$
g_{C M 2}(r)=\left\langle\delta\left(r-R_{C M, i j}\right)\right\rangle, \quad R_{C M, i j}=\left|\mathbf{R}_{C M, i}-\mathbf{R}_{C M, j}\right|,
$$

where the $i$-centroid position vector is $\mathbf{R}_{C M, i}=P^{-1} \sum_{t} \mathbf{r}_{i}^{t}$. The continuous linear response pair function $g_{L R 2}(r)$ is not utilized as a general tool in this work, ${ }^{30,32,54}$ since for solid phases, apart from the slight penetration into the hard core, it is very close to $g_{E T 2}(r)$. Only a couple of examples of $g_{L R 2}(r)$ will be given below to illustrate this latter point.

b) The configurational structure factor given by

$$
S^{C M}(\mathbf{k})=N_{S}^{-2}\left|\sum_{i=1}^{N_{S}} \exp \left(i \mathbf{k} \cdot \mathbf{R}_{C M, i}\right)\right|^{2},
$$


which reaches its maximum value -unity- for certain wave vectors of the perfect solid lattices. ${ }^{55,56}$ For computational convenience, a rectangular parallelepiped $\left(L_{x}, L_{y}, L_{z}\right)$ is normally taken as the central box in simulation work, regardless of the lattice type. ${ }^{8}$ Therefore, wave vectors are expressed in terms of the three side lengths as $\mathbf{k}=2 \pi\left(k_{x} / L_{x}, k_{y} / L_{y}, k_{z} / L_{z}\right)$, where $k_{x}, k_{y}$, and $k_{z}$ are integers. Sets of three commensurate wave vectors can be selected for perfect lattices so as to have $\mathrm{e}^{55}$

$\left|\mathbf{k}_{1} \cdot\left(\mathbf{k}_{2} \times \mathbf{k}_{3}\right)\right|=N_{S}(2 \pi)^{3} / V_{S}$,

which in the case of cubic lattices the three of them reach $S_{\max }=1$, whereas in the hcp case one can always find one wave vector associated with the parallelepiped for which $S_{\max }=1$. If the final phase attained in a simulation admits sets of commensurate wave vectors (although with $S_{\max }<1$ ), this will be indicative of its closeness to the standard lattice types. Hereafter, the values of $S^{C M}$ will be also referred to as the intensities of the wave vectors $\mathbf{k}$.

c) Steinhardt et al $Q_{4}^{C M}$ and $Q_{6}^{C M}$ parameters, which are given by the rotationally invariant configurational quantities ${ }^{57}$

$$
Q_{l}^{C M}=\left\{\frac{4 \pi}{2 l+1} \sum_{m=-l}^{l}\left|\frac{1}{N_{b}} \sum_{b} Y_{l m}\left(\theta_{C M, i j}, \phi_{C M, i j}\right)\right|^{2}\right\}^{1 / 2}, \quad l=4,6 .
$$

The $b$-sum runs over all the fictitious bonds between pairs of centroids separated by less than a given cut-off distance $d_{C}$, which insures that around a given centroid all its neighbors in the first coordination shell are counted. The quantities $\theta$ and $\phi$ are the polar angles of $\mathbf{R}_{C M, i}-\mathbf{R}_{C M, j}$ with respect to a reference coordinate system. The set of $Q_{l}$ parameters take well-defined values for perfect lattices, thus serving as a complementary basis of comparison for identifying solid phases in simulations. Table I shows the perfect lattice values of $Q_{4}$ and $Q_{6}$ for $f c c, b c c$ and $h c p$, together with examples of sets of commensurate wave vectors which maximize $S(\mathbf{k})$. It is 
worthwhile to note that for partially crystalline phases the qualitative behaviors of all the ET2 (or LR2) parameters follow those of CM2, although the latter show more pronounced features. ${ }^{30,31}$

\section{COMPUTATIONAL DETAILS}

PIMC simulations were carried out in the canonical ensemble for the $f c c, b c c$ and $h c p$ lattices. The ranges of $\left(\rho_{N}^{*}, \lambda_{B}^{*}\right)$ conditions explored were as follows. For the $f c c$ and $h c p$ lattices: $\left(0.8 \leq \rho_{N}^{*} \leq 0.9 ; \lambda_{B}^{*}=0.2\right), \quad\left(0.68 \leq \rho_{N}^{*} \leq 0.8 ; \lambda_{B}^{*}=0.4\right), \quad\left(0.6 \leq \rho_{N}^{*} \leq 0.7 ; \quad \lambda_{B}^{*}=0.6\right)$, $\left(0.525 \leq \rho_{N}^{*} \leq 0.625 ; \lambda_{B}^{*}=0.8\right)$, using for most of the computations the density spacing $\Delta \rho_{N}^{*}=$ 0.025 (also $0.02,0.03$ and 0.05 ). For the bcc lattice: $\left(0.8125 \leq \rho_{N}^{*} \leq 0.925, \lambda_{B}^{*}=0.2\right)$, $\left(0.69 \leq \rho_{N}^{*} \leq 0.825 ; \lambda_{B}^{*}=0.4\right),\left(0.6 \leq \rho_{N}^{*} \leq 0.725 ; \lambda_{B}^{*}=0.6\right),\left(0.5375 \leq \rho_{N}^{*} \leq 0.675, \rho_{N}^{*}=0.9 ;\right.$ $\lambda_{B}^{*}=0.8$ ), using $\Delta \rho_{N}^{*}=0.0125,0.02$ and 0.025 . The sample sizes employed $N_{S} \times P$ were taken as: a) $500 \times 12$ for $f c c$; b) $512 \times 12$ for $h c p$; and c) $432 \times 12$ for $b c c$ [also $432 \times 24$ at $\left.\left(\rho_{N}^{*}=0.9 ; \lambda_{B}^{*}=0.8\right)\right]$. The central simulation boxes were taken as cubes $(f c c$ and $b c c)$ or right parallelepipeds $(h c p)$, and the usual periodic boundary conditions were employed. The corresponding perfect lattice was always selected as the initial configuration at every state point for $f c c, h c p$ and $b c c$. In addition to this, $b c c$ complementary compression (increasing-density) runs were conducted along each isotherm in order to clarify finer details of the final solid structures obtained. In these $b c c$-compression calculations, the last configuration obtained for a state point at a given density was used as the starting point for the simulation of the state point at the next (higher) density by rescaling the bead coordinates. By using a density spacing $\Delta \rho_{N}^{*}=0.0125$, the five state points analyzed per isotherm within this compression program were the following: a) along $\lambda_{B}^{*}=0.2$, starting from $\rho_{N}^{*}=0.8375,0.85 \leq \rho_{N}^{*} \leq 0.9 ;$ b) along $\lambda_{B}^{*}=0.4$, 
starting from $\rho_{N}^{*}=0.725,0.7375 \leq \rho_{N}^{*} \leq 0.7875$; c) along $\lambda_{B}^{*}=0.6$, starting from $\rho_{N}^{*}=0.635$, $0.65 \leq \rho_{N}^{*} \leq 0.7 ;$ d) along $\lambda_{B}^{*}=0.8$, starting from $\rho_{N}^{*}=0.575,0.5875 \leq \rho_{N}^{*} \leq 0.6375$.

In the EC applications to the $f c c$ and $h c p$ lattices (Table II) the target points selected were the same: $\left(\rho_{N}^{*}=0.8 ; \lambda_{B}^{*}=0.2\right),\left(\rho_{N}^{*}=0.7 ; \lambda_{B}^{*}=0.4\right),\left(\rho_{N}^{*}=0.6 ; \lambda_{B}^{*}=0.6\right),\left(\rho_{N}^{*}=0.6 ; \lambda_{B}^{*}=0.8\right)$. The sample sizes employed were those quoted above, and a number of extra runs were carried out at $h c p\left(\rho_{N}^{*}=0.7 ; \lambda_{B}^{*}=0.4\right)$, with $\left(P=12, N_{S}=64,216\right)$ and $\left(N_{S}=512, P=3,6,18,24\right)$. It is worth remarking that all the Cao-Berne $P=12$ results (mechanical and thermal) reported in this article are fully consistent with those obtained in Ref. 15 by Runge and Chester. The latter authors carried out EC simulations with the simpler image propagator ${ }^{22}$ and $P=40$ for $\lambda_{B}^{*}>0.88$, and did not use explicit COM corrections. All of this indicates the reliability of the all-purpose $P=12$ selection made in this article, and also of Eq. (8).

The particle mass and diameter were set to $m=28.0134$ amu and $\sigma=3.5 \AA$, respectively. The algorithms employed in the PIMC and EC-COM simulations were based on the PI-necklace normal modes along the lines described elsewhere. ${ }^{31}$ After equilibration, the run lengths were as follows ( 1 kpass $=10^{3} N_{S} \times P$ attempted bead moves; standard 50\% - acceptation criterion). a) For the $f c c$ and $h c p$ simulations (PIMC and EC) 800 kpasses, and also in between 1850 and 6250 kpasses for some EC-hcp applications at $\left(\rho_{N}^{*}=0.7 ; \lambda_{B}^{*}=0.4\right)$. b) For the $b c c$ simulations (PIMC) 1840 kpasses at each state point, augmented to 2760 kpasses at some of them (e.g. lowest densities along each isotherm, and the $P=24$ calculation). Subaverages for mechanical properties ( $E, p$, and $g_{2}$ functions) were calculated with blocks sized 80 kpasses ( $f c c$ and $h c p$ ) and 92 kpasses $(b c c)$. In this connection, to compute $g_{E T 2}$ and $g_{C M 2}$ the total number of configurations employed were $8 \times 10^{4}$ for $f c c$ and $h c p$, and in between $18.4 \times 10^{4}-27.6 \times 10^{4}$ for $b c c$. These configurations were taken at regular intervals throughout the corresponding simulation. Histograms for these functions were defined with $0.1 \AA$ as the width of the bins, 
except for the short-range distances of $g_{E T 2}$ in the vicinity of the hard core, where bins of 0.005 $\AA$ and $0.01 \AA$ were used. ${ }^{49}$ This yields the centers of the bins for distances (in $\AA$ ) not close to the hard core as follows: a) for ET2, $(3.65,3.75,3.85$, etc.); and b) for CM2, $(3.45,3.55,3.65$, etc.). For uniformity of comparison with the perfect lattice positions of neighboring particles around a given one, in the graphs below the latter histogram centers were used as the relative positions of neighbors in the perfect lattice (differences from the actual positions are negligible). Also, for convenience of further reference, the distances will be reported in the figures in reduced units, i.e. $r^{*}=r / \sigma$.

Solid pressures were computed by making use of the behavior $g_{E T 2}(r)=a(r-\sigma)^{2}$ in the vicinity of the hard core, ${ }^{11(b), 13}$ and applying the weighting procedure described in Ref. 49. To obtain the reversible work $A_{2} / R T$ in Eq. (8) the EC maximal parameters $\alpha_{M}$ and the auxiliary constants $\left(c_{0}\right)^{-1}=\left\langle\left(\mathbf{r}-\mathbf{r}_{0}\right)^{2}\right\rangle_{\alpha_{C}=0}$ also had to be determined, which was done along the lines explained in Ref. 31. These EC basic parameters are collected in Table II, where one can observe that the assumption of $\alpha_{M}(P) \sim P$ seems to be close to the true dependence. Gauss-Legendre quadrature with ten points was used to compute $A_{2} / R T$. The $\alpha_{M}$ values chosen render $W_{N P}$ lower than $2 \%$ of the whole effective potential in Eq. (6), and the actual $W_{N P}$ contribution in Eq. (8) was fixed with umbrella sampling. The $\left(\mathbf{r}_{i}^{t}-\mathbf{r}_{0, i}\right)^{2}$ statistics were gathered every 5000 attempted normal-mode moves (also 2000 moves for the $N_{S}=64,216$ hcp test simulations). The reader is referred to Ref. 31 for full details regarding all these calculations. Except for the foregoing Gauss-Legendre quadrature applications, numerical integrations in this article were performed using an algorithm based on cubic splines.

Calculations of the order parameters $Q_{4}^{C M}, Q_{6}^{C M}$ and $S^{C M}(\mathbf{k})$ were carried out three times per block at equally spaced intervals. Although this scanning of parameters is not 
exhaustive, it keeps the cost of the simulations low and, in all likelihood, produces uncorrelated data regarding the structure to be analyzed. Besides, it turns out to be sufficient to determine the salient structural traits of the solid phases. Note that the CM2 parameters serve to better identify the symmetries obtained ${ }^{30,31}$ than if the ET2 parameters had been used instead. The $Q-$ neighborhood parameter $d_{C}$ was set to $1.5 \sigma$ (also $2 \sigma$ at low densities along $\lambda_{B}^{*}=0.8$ ). The values reported below for $Q_{4}^{C M}$ and $Q_{6}^{C M}$ are the resulting averages over the runs. As regards $S^{C M}(\mathbf{k})$, the ten $\mathbf{k}$ wave vectors leading to the largest intensities per block were recorded. To do so, triplets of integers $\left(k_{x}, k_{y}, k_{z}\right)$ in the mesh $25 \leq k_{x}^{2}+k_{y}^{2}+k_{z}^{2} \leq 100,-10 \leq k_{i} \leq 10$, were monitored. This allows one to estimate the limits of variation of this quantity in partially crystalline phases.

The determination of the fluid-solid $(f c c, h c p)$ coexistence was achieved at each isotherm by finding the intercept between the fluid and solid $G=G(p)$ curves. The statistical errors in the common $(p, G)$ were obtained by adding the variances of each property at the corresponding fluid and solid state points. These errors have been employed to define those affecting the coexistence densities.

\section{RESULTS}

\section{A. $f c c$ and $h c p$ mechanical properties}

Figures 1 and 2 show the radial correlation functions obtained at typical $f c c$ and $h c p$ state points. As seen, the positions of the maxima and minima agree very well with the expected for the perfect lattices. Although well-known, the $f c c / h c p$ differences beyond the third peak have to be kept in mind for a better understanding of the $b c c$ results obtained in this work. Also, the close proximity between $g_{E T 2}$ and $g_{L R 2}$ is apparent. In addition to this, sets of three commensurate wave vectors that maximize $S^{C M}(\mathbf{k})$ have been found. They agree with those which arise in the 
$f c c$ and $h c p$ perfect lattices. The maximal $S_{\max }^{C M}(\mathbf{k})$ increase with the density and, for example along the two extreme isotherms, are in between the following values: ${ }^{58}$ a) for $f c c$, $\left(0.69 \leq S_{\max }^{C M} \leq 0.87 ; \lambda_{B}^{*}=0.2\right) \quad$ and $\quad\left(0.66 \leq S_{\max }^{C M} \leq 0.87 ; \lambda_{B}^{*}=0.8\right) ; \quad$ and $\quad$ b) $\quad$ for $\quad h c p$, $\left(0.68 \leq S_{\max }^{C M} \leq 0.84 ; \lambda_{B}^{*}=0.2\right)$ and $\left(0.64 \leq S_{\max }^{C M} \leq 0.87 ; \lambda_{B}^{*}=0.8\right)$

The mean centroid parameters $Q_{4}^{C M}$ and $Q_{6}^{C M}$ behave in accord with the foregoing results. For example, the mean values found along the two extreme isotherms are: ${ }^{58}$ a) for $f c c$, $\left(0.13 \leq Q_{4}^{C M} \leq 0.16 ; 0.40 \leq Q_{6}^{C M} \leq 0.49 ; \lambda_{B}^{*}=0.2\right)$ and $\left(0.15 \leq Q_{4}^{C M} \leq 0.18 ; \quad 0.41 \leq Q_{6}^{C M} \leq 0.52 ;\right.$ $\left.\lambda_{B}^{*}=0.8\right) ; \quad$ and $\quad$ b) $\quad$ for $h c p, \quad\left(0.07 \leq Q_{4}^{C M} \leq 0.08 ; 0.34 \leq Q_{6}^{C M} \leq 0.42 ; \quad \lambda_{B}^{*}=0.2\right) \quad$ and $\left(0.08 \leq Q_{4}^{C M} \leq 0.09 ; \quad 0.35 \leq Q_{6}^{C M} \leq 0.44 ; \quad \lambda_{B}^{*}=0.8\right)$. The overall agreement between the patterns shown by all the order parameters indicates the internal consistency of the present calculations. Therefore, all the solid phases obtained in these groups of simulations can be safely classified as partially crystalline $f c c$ or $h c p$.

The comparison between the current results for $f c c$ and those reported in Ref. 31 reveals that: i) state points $\left(\rho_{N}^{*}=0.775 ; \lambda_{B}^{*}=0.2\right)$ and $\left(\rho_{N}^{*}=0.58 ; \lambda_{B}^{*}=0.6\right)$, formerly classified as solid phases, have long equilibration stages and now are proven to be fluid; and ii) for the rest of the state points, $S_{\max }^{C M}(\mathbf{k}), Q_{4}^{C M}$ and $Q_{6}^{C M}$ do not differ significantly, and this in spite of the much less demanding sampling carried out in Ref. 31.

Figures 3 and 4 show the $f_{c c}$ and $h c p$ mean reduced internal energies $E / R T$ and mean compression factors $P V / R T$. Also shown are the fluid branches along each isotherm, as determined in Ref. 49 with isothermal compressibility data. The numerical results are given as supplementary material. ${ }^{58}$ The statistical error (one-standard deviation) in the solid phase values for $E / R T$ and $P V / R T$ remains below $0.5 \%$ and $1.7 \%$, respectively. Within these margins no significant $f c c / h c p$ differences appear at any state point. This raises the question of the possibility of detecting the influence of the different packing patterns on the relative stability of the $f c c$ and 
hcp quantum lattices. The consideration of this interesting issue, which has its counterpart in classical statistical mechanics, ${ }^{7}$ is deferred to subsection C.

\section{B. Features of the EC calculations}

To grasp the salient features of the EC calculations Table III contains the results at hcp $\left(\rho_{N}^{*}=0.7 ; \lambda_{B}^{*}=0.4\right)$ using different sample sizes and run lengths. All the behaviors discussed in Sec. II.B are present here. For instance: i) the increasing absolute values of $A_{1}$ and $A_{2}$ with $P$, their compensation, and the effect of the umbrella sampled $A_{3}$; ii) the role of the COM corrections coming from $A_{4}$ and $A_{5}$, which become smaller (or larger) with increasing $N_{S}$ (or $P)$ - also recall that $A_{4}=f\left(\alpha_{M}\right)$-; iii) the $N_{S}$ - control on the $P$ effect; and iv) the scale of the computational effort to achieve higher accuracy in the determination of $A_{2}$, and thereby of $A$ $\left(512 \times 24\right.$ results). As seen in Table III, when the proposed COM corrections ${ }^{31}$ are used in Eq. (8) they bring about fully consistent results for the different sample sizes employed. This latter effect is more noticeable for low values of the $N_{S}$ or $P$ parameters. As seen, practical convergence is reached by increasing the sample size and the run length. All of this is very rewarding in that the current EC calculations of QHS free energies do not yield any irregular behavior in the results.

With respect to the effect of applying the classical expression for $A_{5} / R T$ instead of that given in Eq. (8), it is worth noting the following facts. First, the changes in $A / R T$ turn out to be even smaller than the final statistical errors. For example: a) the most extreme case occurs at the least physically significant sample $64 \times 12$, for which $A / R T=1.588(74) \rightarrow 1.607(74)$; and $b$ ) the latter situation should be compared with that of the sample $512 \times 12$, for which $A / R T=1.655(59) \rightarrow 1.658(59)$. Second, although not shown in Table III, in this regard the results for the rest of the target state points calculated are similar, for example at $f c c-500 \times 12$ $\left(\rho_{N}^{*}=0.6 ; \lambda_{B}^{*}=0.8\right) \quad A / R T=5.576(41) \rightarrow 5.579(41)$. 


\section{C. $f c c$ and $h c p$ - fluid equilibria}

Table IV contains the thermodynamic data of the fluid-solid coexistence as obtained in this work. The slight differences between $f c c$ and $h c p$ can be explained by the error bars in the results. Therefore, no significant differences between both solid-fluid equilibria have been obtained. In particular, the $f c c$ and $h c p$ relative stabilities along their corresponding coexistence lines with the fluid phase cannot be distinguished from one another. The same result is obtained when comparing the relative stabilities of the $f c c$ and $h c p$ state points along the four isotherms. ${ }^{58}$ In Table IV the pressure is reported in reduced units $p^{*}=p /\left(\hbar^{2} m^{-1} \sigma^{-5}\right)$, whereas Gibbs energy is reported in units of $R T$, as usual.

In this connection, through extensive and thorough computations, ${ }^{7}$ it was found some years ago that in the classical hard sphere (CHS) system the $f c c$ lattice is more stable than the hcp lattice, but by a really small fraction of $R T$ (i.e. for $A$-energy: from $0.26 \%$ at close packing to $0.23 \%$ at melting). Nevertheless, the same sort of question in the QHS system still seems far from being settled. A number of issues should be addressed in undertaking this project. Among them one may mention: i) the computation of solid pressures with more elaborate procedures than the based on Eq. (2) and/or the conducting of simulations in the $(N, P, T)$ ensemble ${ }^{59}$ to complement the thermodynamic data on this system; ii) the use of more advanced pair actions, ${ }^{24}$ iii) the going beyond pair actions to incorporate many-body effects; and iv) the solving of the geometry problems associated with the fixed-COM corrections. ${ }^{53}$ Note that quantum hard spheres repel each other before contact, the repulsion being strongly dependent on the second derivative at contact of $g_{E T}$ [Eq. (2)]. Hence, some sort of concerted many-body behavior could play a significant role as the density increases. This may be regarded as a situation parallel to that observed in CHS: significant $f c c / h c p$ differences in the pressure were identified as arising from collision contributions associated with successive-shell neighbors. ${ }^{3,7}$ In addition to this, there is the problem of the computational efficiency. In this regard, the QHS non-analytic interactions 
make it difficult to devise alternatives (e.g. Morales-Singer, ${ }^{52(e), 60}$ PI molecular dynamics) ${ }^{61}$ different from the general Monte Carlo one followed in this article.

Another point to be considered is the comparison with the former $f c c$-fluid equilibrium results based on a previous calculation of the fluid isothermal compressibilities. ${ }^{31}$ There are a number of dramatic changes (Figs. 5 and 6) which are to be ascribed to the much better general accuracy achieved both in this work and in Ref. 49, and also to the correct identification of the fluid state points mentioned above. In general, the coexistence properties $\rho_{N}^{*}$ (fluid and solid), $p$, and $G$, are shifted to higher values. The most pronounced changes occur at $\lambda_{B}^{*}=0.2$ and 0.6 , for which the shifts in $(p, G)$ are $(15 \%, 16 \%)$ and $(26 \%, 22 \%)$, respectively. Note the logarithmic scale used for pressures in Fig. 5. Moreover, the anomalous behavior of the entropy ${ }^{31}$ on both sides of the coexistence line at $\lambda_{B}^{*}=0.4$ and 0.6 is not present in the current calculations. Thus, clear monotonic patterns for $G / R T$ and $S / R$ with $\lambda_{B}^{*}$ are obtained (Fig. 6). Furthermore, the fractional change in density $\Delta \bar{\rho}=\left(\rho_{S}-\rho_{F}\right) / \rho_{S}(\mathrm{~S}=$ solid; $\mathrm{F}=$ fluid $)$ is modified and indicates a larger separation from the classical value $\mathrm{e}^{9,62}(0.094-0.095)$ at $\lambda_{B}^{*}=0.2$, that is: 0.091 (former) ${ }^{31}$ $\rightarrow 0.086$ (Table IV). It is also interesting to remark that the linear dependence between the fluid and the solid densities along coexistence is still shown by the current results. By extending these results with the classical data, ${ }^{62}$ plus Runge-Chester's results ${ }^{15}$ at $\lambda_{B}^{*}=0.89,1.25,1.98$, one finds again an almost perfect linear correlation between the mean densities $\rho_{f c c}^{*}=1.143 \rho_{F}^{*}-0.038$ (correlation coefficient $=0.99997)$. There still remains a valuable extra feature related to the absolute maxima of the fluid centroid structure factors along the coexistence line. ${ }^{49}$ The rather irregular behavior obtained in Ref. 32 is absent from the current calculations. As determined in the present work, these $S^{C M}(\mathbf{k})$ maximal intensities fall within $\approx 3 \pm 0.06$, a uniformity which is 
to be compared with the general classical result $2.85 \pm 0.1$, which also holds for classical hard spheres. $^{63}$

Under the current conditions, and in spite of the substantial differences between QHS and CHS, one obtains for the entropy of both changes of phase $\Delta S_{F \rightarrow S}^{Q H S} / R \approx-1.1 \pm 0.1$, which is certainly close to the classical value ${ }^{9,63} \Delta S_{F \rightarrow S}^{C H S} / R \approx-1.16$. In addition, note the smallness of the energy differences between the fluid and solid phases at coexistence. Therefore, the quantum changes of phase in Table IV are controlled by entropic effects, which is the same to what is observed in the classical domain. Moreover, as observed in Ref.15, to find the energy-controlled regime in QHS one should go to very low temperatures $\left(\lambda_{B}^{*}>2\right)$ and deal with quantum statistics for the fluid phase.

Figure 7 shows a comparison between the melting-freezing lines of QHS and ${ }^{4} \mathrm{He}$ for $\lambda_{B}^{*}<2$, where the experimental data have been taken from McCarty's work ${ }^{64}$ and the value $\sigma\left({ }^{4} \mathrm{He}\right)=2.2 \AA$ has been assumed. ${ }^{15}$ As seen, the patterns are consistent with each other, which is in agreement with the well-known image of helium atoms as hard spheres. The differences can be fixed by adding an attractive tail to the hard-sphere interaction and using perturbation theory, as discussed in Ref. 15, although to complete such a project a further definition of an effective collision parameter as $\sigma\left(\rho_{N}^{*}, \lambda_{B}^{*}\right)$ should be made.

\section{The peculiar bcc-q solids}

Figure 8 shows the energy and the compression factor for most of the final $b c c$-q state points investigated by starting their simulations from the corresponding $b c c$-perfect lattices (related data are given in the supplementary material). ${ }^{58}$ At each isotherm one can observe that both quantities display some sort of irregular behavior, which for the reader to grasp is hinted approximately by dotted segments in Fig. 8. As seen, the effect is more pronounced in the compression factor. This effect suggests the possibility of two solid branches for the $b c c$-q data. 
The inclusion of state points at higher densities would even reveal the possibility of a third solid branch, but the following analysis will concentrate just on Fig. 8 .

On the other hand, the route based on the $b c c$-compressions yields thermodynamic (and structural) results that, in the overwhelming majority of cases, agree within statistics with those in Fig. 8. This has been determined at a level of significance 5\% (two-sided) by assuming Gaussian behavior. Only at $\left(\rho_{N}^{*}=0.8625 ; \lambda_{B}^{*}=0.2\right)$ and $\left(\rho_{N}^{*}=0.6625 ; \lambda_{B}^{*}=0.6\right)$ the $E / R T$ and $P V / R T$ mean values are greater than, although relatively close to, those of their corresponding state points in Fig.8. These differences cannot be explained by statistics, and this suggests that by reducing further the density spacing in the compression program they could be fixed, and an even better agreement between the solids obtained via both routes could be obtained. Nevertheless, the whole assessment of this question, such as is, cannot be regarded as conclusive whatsoever. At this juncture, and to avoid carrying out more computations, one can resort to a detailed analysis of the structural parameters obtained in the $b c c$-q simulations.

First, $Q_{6}^{C M}$ always remains lower than the perfect lattice value $(0.511)$ along every isotherm, whilst for $\lambda_{B}^{*}>0.2 Q_{4}^{C M}$ can reach values that are significantly higher than its reference (0.036). ${ }^{57}$ Thus, one finds $Q_{4}^{C M}$ to be in between: a) $0.045-0.056$ at $\lambda_{B}^{*}=0.4$; b) $0.057-0.089$ at $\lambda_{B}^{*}=0.6$; and c) $0.020-0.111$ at $\lambda_{B}^{*}=0.8$. Moreover, the variations in the $Q^{C M}$ parameters do not appear to be always monotonic with the density. ${ }^{58}$ These facts reveal that the mechanically stable solids attained can be far from being proper $b b c$ lattices. In this regard, one should bear in mind the $f c c$ and $h c p$ reference values given in Table I, in particular $Q_{4}^{C M}(h c p)=$ 0.097 , and $Q_{4}^{C M}(f c c)=0.191$.

Second, despite the fact that sets of $b c c$-commensurate maximizing wave vectors Eq. (11) are always obtained, the k-space results for the PI centroids along the four isotherms point to important changes in the original structure of the $b c c$-lattice (Table V and supplementary 
material). ${ }^{58}$ The situation may be summarized as follows. i) For low densities the intensities of the three maximizing wave vectors are far from unity, but they remain close to each other, which is the expected result. ii) As the density increases, the maximum intensity increases with respect to the latter set. At the same time, one finds a range of medium densities in which the three intensities separate significantly from each other. This anomalous effect is noteworthy in that one of the intensities becomes much smaller than the other two. And, iii) by increasing further the density, the intensity values close up again reaching higher values than those in i) and ii). This indicates that the resulting solid phase at high density has reorganized itself into a more ordered structure. To illustrate the latter feature only some significant data of the simulations at $\left(\rho_{N}^{*}=0.925 ; \lambda_{B}^{*}=0.2\right)$ and $\left(\rho_{N}^{*}=0.9 ; \lambda_{B}^{*}=0.8\right)$ are reported in Table $\mathrm{V}$, where one observes that the centroid intensities reach maximal typical values 0.75 and 0.78 , respectively. All of this $b c c$-behavior contrasts sharply with those of $f c c$ and $h c p$ (Table V). Therefore, along every bcc isotherm there are three ranges of densities (low, medium, and high), each of which showing a distinctive structure. This amounts to having three types of different phases, which will be termed $b c c$-q: $b c c$-qI for low densities, $b c c$-qII for medium densities, and $b c c$-qIII for high densities. However, the limitations imposed by the periodic boundary conditions used in the $b c c$ simulations preclude one from drawing further conclusions from the $\mathbf{k}$-space data.

Third, one notes that the openness of the bcc lattice has to play a role in the above behavior (e.g. the $b c c$ packing fraction is 0.68 , whereas that of $f c c$ or $h c p$ is 0.74 ). Two QHS additional features are to be taken into account: the really strong repulsions and the quantum delocalization of particles. Therefore, as the density increases a variety of subtle arrangements of the particles can occur yielding the so-called $b c c-q$ solids. ${ }^{30}$ This discussion can be complemented by inspecting some typical shapes of the pair radial correlations. For example Fig. 9, where two facts are to be noted: i) that the proper $b c c$ symmetry is not contained in the examples shown; and b) that the CM2 and ET2 salient features coincide with one another (CM2 
is obviously more structured). As a matter of fact, and in agreement with the foregoing $b c c-q$ classification based on k-space data, one finds in the obtained results the following three different types of solid radial structures. i) $b c c$-qI, which is similar to that of $b c c$ classical calculations. ${ }^{34,36}$ This is the type of structure which has been taken as the starting point for the increasing-density program along isotherms. ii) $b c c$-qII, which corresponds to the lower plots in Fig. $9\left(\rho_{N}^{*}=0.9 ; \lambda_{B}^{*}=0.2\right)$. And iii) $b c c$-qIII, which corresponds to the upper plots in Fig. 9 $\left(\rho_{N}^{*}=0.9 ; \lambda_{B}^{*}=0.8\right)$.

Fourth, the comparison between the $b c c-\mathrm{q}$ and the $h c p$ pair radial correlation functions yields a surprising result: within the $b c c$-qII range of conditions the perfect- $b c c$ lattice adjusts itself to perform a spontaneous transition to an $h c p$-like lattice, i.e. $b c c \rightarrow b c c$-qII $\approx h c p$. With a few exceptions, this sort of transition takes place quickly, after two or three equilibration blocks. Although this result can be seen in the graph for the state point at $\lambda_{B}^{*}=0.2$ in Fig. 9, Figs. 10 and 11 describe this situation in a more complete way. Figure 10 displays the marked similarity between $b c c$-qII and $h c p$ at another representative state point $\left(\rho_{N}^{*}=0.625 ; \lambda_{B}^{*}=0.8\right)$. Figure 11 contains the plots of the ET2 functions at the latter state point and shows, beyond any reasonable doubt, the closeness between the $b c c$-qII and $h c p$ actual structures (instantaneous) and, also, the patent dissimilarities between $b c c$-qII and $f c c$. Also, $h c p$ presents peaks and valleys slightly more pronounced than $b c c$-qII $\left(r^{*} \leq 4\right.$ or $r / \AA \leq 14$ for the parameters used in this work). This behavior occurs along the four isotherms investigated, and it displays clearly the quantum phase transition $b c c \rightarrow h c p$. The following results are worth noting: a) the $b c c-\mathrm{q} Q_{4}^{C M}$ values can become quite close to that of perfect $h c p(0.097)$; and b) although $Q_{4}^{C M}$ is more sensitive than $Q_{6}^{C M}$ to identifying these lattice similarities, for certain $\left(\rho_{N}^{*}, \lambda_{B}^{*}\right)$ conditions the $b c c$-qII and the $h c p$ structures show a remarkable proximity between their $Q^{C M}$ order parameters. ${ }^{58}$ 
The $f c c$ and $h c p$ structures do not present any of the $b c c$-q problems (Table V, Figs. 1 and 2, and Ref. 58), which is what permits devising safe EC procedures to compute QHS free energies for these regular quantum lattices. However, the above $b c c$-q features pose major symmetry problems to the calculation of QHS free energies. Extreme examples are, for instance, the density conditions for which $b c c$-qII and $b c c$-qIII state points are obtained, for the definition of a significant reversible path leading to a perfect $b c c$ Einstein crystal is not feasible. It is interesting to recall that, when dealing with classical hard spheres arranged in a bcc lattice (a system whose structure is unstable to shear), a useful device to carry out some exploratory freeenergy calculations is to impose within the simulation cell constraints that maintain the integrity of this lattice. ${ }^{34}$ Clearly, the results of this procedure cannot lead to a true thermodynamically stable $b c c$ phase.

The energies and the pressures of $b c c$-q phases are significantly higher than those of the $f c c$ or $h c p$ phases at the same density and temperature. This fact and the $b c c$ structural problems point to the thermodynamic instability of $b c c$-q with respect to the close-packed structures. With regard to the comparison between the energies and pressures of $b c c-q I I$ and $h c p$, it is easy to understand that the differences result from the combination of the QHS behavior and the unnatural space (i.e. a cube instead of a right parallelepiped) in which the simulated $b c c$-qII phases are trapped.

Finally, in relation to the two-way computations performed, the direct $b c c \rightarrow b c c-$ II $\approx$ $h c p$ and the compression $b c c-\mathrm{qI} \rightarrow\{b c c-\mathrm{qII} \approx h c p\}$, a reconsideration of the features present in Fig. 8 is in order. When differences in the phase entropies are expected (e.g. the fluid-solid transitions in Table IV), one may apply the rationale that QHS phase transitions for $\lambda_{B}^{*} \leq 0.8$ should be entropy-driven. In this way, the really small irregularities in the $b c c$-q energies might be explained. However, a better explanation can be provided by noting that this phase transition shows signatures of a second-order transition. First, the calculations $b c c \rightarrow b c c$-qII show that at 
constant volume there are direct changes in the solid symmetry (Fig.12), a fact supported further by the consistency with the results arising from the $b c c-\mathrm{qI} \rightarrow\{b c c-\mathrm{qII}\}$ calculations. Second, no discontinuity in the interaction potential energy is present. Accordingly, one can expect a continuous behavior of $E / R T$ and of $P V / R T$ at the transition points in the isotherms investigated (the same for the rest of thermodynamic properties). On the other hand, given that a finite density spacing is employed, and also that the pressure is more sensitive to the short-range structure of the solid phase under consideration [i.e. Eq. (2)], the results display a more pronounced irregularity in $P V / R T$ than in $E / R T$ on both sides of the transition.

\section{CONCLUSION}

In this study of quantum hard sphere solids $(f c c, h c p, b c c)$ mechanical, structural and thermal properties have been determined using path integral Monte Carlo simulations involving Cao-Berne pair action and also the Einstein crystal technique. ${ }^{31}$ An extensive use of centroid quantities $\left[Q_{4}^{C M}, Q_{6}^{C M}, g_{C M 2}(R)\right.$, and $\left.S^{C M}(\mathbf{k})\right]$, and also of the instantaneous function $g_{E T 2}(r)$, has been made to characterize all the solid phases obtained in this work. As expected, the $g_{E T 2}(r)$ instantaneous features follow closely those of $g_{C M 2}(R)$. Although the $S^{C M}(\mathbf{k})$ information has been in general very useful, in the $b c c$ applications the symmetry problems and the restrictions imposed by the periodic boundary conditions do not allow one to interpret the related $\mathbf{k}$-space results in a direct way.

The simulations to obtain mechanical $(f c c, h c p$ and $b c c)$ and thermal properties $(f c c$ and $h c p$ ) have employed long run lengths, thereby yielding more accurate results than in previous works. $^{30-32}$ These more accurate results, together with the improved equation of state for the fluid phase reported in Ref. 49, have produced reliable results for the fluid - solid ( $f c c$ or $h c p$ ) equilibria. In this regard, no significant differences between both equilibria have been obtained.

Along the two isotherms $\lambda_{B}^{*}=0.2$ and 0.6 , some of the former results regarding the fluid $-f c c$ 
equilibrium have undergone significant changes. For example, there is an increase in the coexistence pressure and Gibbs energy by an amount in between $15 \%-26 \%$. Also, a uniform behavior of the main peak of the centroid structure factors $(\approx 3.0)$ at the fluid side of the coexistence line is obtained. Moreover, it is worth noting that, none of the thermodynamic results concerning the fluid-solid coexistence in this article are out of the ranges signaled by the structural calculations. Furthermore, the Einstein crystal technique as developed in Ref. 31 has been shown to provide a useful computational framework for path-integral applications, as it yields consistent results when variations in the simulation sample size $N_{S} \times P$ are made.

In the current $f c c$ and $h c p$ calculations no unforeseen order behavior has been observed. Although the possibility of establishing the relative stability of the $f c c$ and the $h c p$ phases has been considered, no significant differences between them have been found. It is clear that there remains a great deal of work to be done to settle this question, and a number of different complementary strategies have been discussed in the main text of this article. One might hope to find a less expensive and, perhaps, more rigorous method (avoiding the unsolved geometryvolume problems $)^{53}$ to obtain free energies for the stable lattices of PI quantum hard spheres. However, as discussed in this work, the singular nature of the QHS interactions makes it difficult to complete some stages of such a project in a straightforward way.

On the other hand, the theoretical $b c c$ quantum hard-sphere lattice turns out to be mechanically unstable and its integrity is not kept in the simulations. This is analogous to the classical situation, although some important differences have been observed in this study. A first consequence of this instability is that three different types of solid structures have been obtained: $b c c$-qI (classical-like), ${ }^{36} b c c$-qII (hcp-like), and $b c c$-qIII (ordered, but highly irregular). They are far from the perfect bcc symmetry, occur along every isotherm investigated, and their appearance depends on the density of the sample. The most striking fact is that for medium densities the twoway $b c c$ simulations performed lead to spontaneous transitions to $h c p$-like structures ( $b c c$-qII). 
Furthermore, these $b c c \rightarrow b c c$-qII $\approx h c p$ transitions show typical second-order transition signatures, a result which seems to be in sharp contrast with the classical situation. ${ }^{34,36,65}$ In identifying this change in the symmetry of the solid phases, the use of the order parameters has been crucial. One notes that the computation of energies, pressures and structure factors, hinted that different $b c c-\mathrm{q}$ solid phases arose from the simulations. However, clear checks of the spontaneous transitions $b c c \rightarrow h c p$-like (and not $f c c$ !) have been obtained through: i) the pair radial functions (centroid and instantaneous); and ii) the sensitivity of the $Q_{4}^{C M}$ parameter. The computation of free energies for the above $b c c$-q phases seems far from being straightforward owing to the major symmetry problems they present.

Therefore, and despite the absence of attractions, one is forced to conclude that in the QHS system the strong repulsions together with the delocalization of the particles, by finding their way through the voids of the $b c c$ structure, can act as a source of order under certain conditions $\left(\rho_{N}^{*}, \lambda_{B}^{*}\right)$. Accordingly, given the well-established connections between quantum hard spheres and helium atoms for medium and high densities, ${ }^{14-15,54}$ one is led to think that the quantum hard-sphere behavior could also play an important role in the helium $b c c \rightarrow h c p$ transition at low temperature (e.g. in ${ }^{4} \mathrm{He} 1.45<T / K<1.8, \lambda_{B}^{*} \approx 3$ ). ${ }^{38,39}$ On the other hand, attractive interactions must be taken into account when dealing with real systems (at high solid densities also triplet interactions are needed). Furthermore, quantum statistics becomes crucial for describing non-perfect crystals and solid-fluid interfaces at low temperatures. ${ }^{41,66}$ Therefore, the final picture for helium solids is certainly far more complex than the one provided by the QHS model in this work.

There remain a number of QHS interesting issues to be worked out, and the results reported in this article are intended to be a useful basis of comparison for further research on this topic. In particular, the spontaneous $b c c \rightarrow h c p$ transition found in this work seems to deserve further investigation. As regards the PI applications where quantum exchange can be neglected it 
may be well-worth exploring: a) the complementary use of other ensembles with increasing sample sizes; ${ }^{59,67-70}$ b) more exhaustive and refined techniques to analyze order in the sample (e.g. true bead-bead order parameters, ${ }^{30,31}$ functional theories ${ }^{34}$ plus simulation, ${ }^{68-70}$ etc.); and c) the inclusion of attractive interactions, which supplement the QHS model ${ }^{48}$ and stabilize the $b c c$ lattice against shear modes. Also, one can try to gain more insight into the outstanding helium solid-solid problems by using hard spheres. In addition to the foregoing measures, one should: d) evaluate the influence of quantum statistics on the $b c c$ stability and on the solid-fluid interfaces; and e) make a comparison with results obtained with realistic models for the He-He interaction. ${ }^{71,72,73}$

\section{ACKNOWLEDGMENT}

The author wishes to thank Comunidad de Madrid (Spain) for financial support through Project Modelización y Simulación de Sistemas Complejos (P2009/ESP-1691).

\section{References}

${ }^{1}$ W. W. Wood, J. Chem. Phys. 48, 415 (1968); 52, 729 (1970); D. A. Young and B. J. Alder, J. Chem. Phys. 60, 1254 (1974).

${ }^{2}$ N. F. Carnahan and K. E. Starling, J. Chem. Phys. 51, 635 (1969).

${ }^{3}$ B. J. Alder, B. P. Carter and D. A. Young, Phys. Rev. 183, 831 (1969).

${ }^{4}$ W. G. Hoover, D. A. Young and R. Grover, J. Chem. Phys. 56, 2207 (1972).

${ }^{5}$ D. Chandler, Annu. Rev. Phys. Chem. 29, 441 (1978).

${ }^{6}$ D. Frenkel and A. J. C. Ladd, J. Chem. Phys. 81, 3188 (1984).

${ }^{7}$ L. V. Woodcock, Faraday Discuss. 106, 325 (1997).

${ }^{8}$ R. J. Speedy, J. Phys.: Condens. Matter 10, 4387 (1998).

${ }^{9}$ W. G. Hoover and F. H. Ree, J. Chem. Phys. 49, 3609 (1968). 
${ }^{10}$ P. N. Pusey and W. van Megen, Nature (London), 320, 340 (1986).

${ }^{11}$ (a) E. H. Lieb, J. Math. Phys. 8, 43 (1967); ${ }^{(b)}$ S. Y. Larsen, J. Chem.. Phys. 48, 1701 (1968).

${ }^{12}$ P. C. Hemmer, Phys. Lett. A 27, 377 (1968); B. Jancovici, Phys. Rev. 184, 119 (1969).

${ }^{13}$ W. G. Gibson, Mol. Phys. 30, 13 (1975).

${ }^{14}$ M. H. Kalos, D. Levesque and L. Verlet, Phys. Rev. A 9, 2178 (1974); L. Dang and M. Boninsegni, Phys. Rev. B 81, 224502 (2010).

${ }^{15}$ K. J. Runge and G. V. Chester, Phys. Rev. B 38, 135 (1988).

${ }^{16}$ B. J. Yoon, M. S. Jhon and H. A. Scheraga, J. Chem. Phys. 96, 7005 (1992).

${ }^{17}$ R. P. Feynman and A. R. Hibbs, Quantum Mechanics and Path Integrals (McGraw-Hill, New York, 1965).

${ }^{18}$ R. P. Feynman, Statistical Mechanics (Benjamin, Reading, 1972).

${ }^{19}$ J. A. Barker, J. Chem. Phys. 70, 2914 (1979).

${ }^{20}$ D. Chandler and P. G. Wolynes, J. Chem. Phys. 74, 4078 (1981).

${ }^{21}$ B. J. Berne and D. Thirumalai, Annu. Rev. Phys. Chem. 37, 401 (1986); T. E. Markland, J. A. Morrone, B. J. Berne, K. Miyazaki, E. Rabani and D. Reichman, Nature Physics 7, 134 (2011).

${ }^{22}$ J. Jacucci and E. Omerti, J. Chem. Phys. 79, 3051 (1983).

${ }^{23}$ J. Cao and B. J. Berne, J. Chem. Phys. 97, 2382 (1992).

${ }^{24}$ E. de Prunelé, J. Phys. A: Math. Theor. 41, 255305 (2008).

${ }^{25}$ P. Grüter, D. M. Ceperley and F. Laloë, Phys. Rev. Lett. 79, 3549 (1997).

${ }^{26}$ L. M. Sesé and R. Ledesma, J. Chem. Phys. 102, 3776 (1995).

${ }^{27}$ L. M. Sesé and R. Ledesma, J. Chem. Phys. 106, 1134 (1997).

${ }^{28}$ D. M. Ceperley, Rev. Mod. Phys. 67, 279 (1995); M. Boninsegni and D. M. Ceperley, Phys. Rev. Lett. 74, 2288 (1995); E. W. Draeger and D. M. Ceperley, Phys. Rev. B 61, 12094 (2000); S. Moroni, F. Pederiva, S. Fantoni and m. Boninsegni, Phys. Rev. Lett. 84, 2650 (2000); E. W. Draeger and D. M. Ceperley, Phys. Rev. Lett. 90, 065301 (2003). 
${ }^{29}$ S. A. Chin and C. R. Chen, J. Chem. Phys. 114, 3338 (2001); S. Jang, S. Jang and G. A. Voth, J. Chem. Phys. 115, 7832 (2001); J. E. Cuervo, P.-N. Roy and M. Boninsegni, J. Chem.. Phys. 122, 114504 (2005); H. Li, N. Blinov, P.-N. Roy and R. J. Le Roy, J. Chem. Phys. 130, 144305 (2009); K. Sakkos, J. Casulleras and J. Boronat, J. Chem. Phys. 130, 204109 (2009); R. E: Zilich, J. M. Mayrhofer and S. A. Chin, J. Chem. Phys. 132, 044103 (2010).

${ }^{30}$ L. M. Sesé, J. Chem. Phys. 114, 1732 (2001).

${ }^{31}$ L. M. Sesé, J. Chem. Phys. 126, 164508 (2007).

${ }^{32}$ L. M. Sesé and L. E. Bailey, J. Chem. Phys 126, 164509 (2007).

${ }^{33}$ S. Alexander and J. McTague, Phys. Rev. Lett. 41, 702 (1978).

${ }^{34}$ W. A. Curtin and K. Runge, Phys. Rev. A 35, 4755 (1987).

${ }^{35}$ H. H. Sample and C. A. Swenson, Phys. Rev. 158, 188 (1967).

${ }^{36}$ D. Levesque, J. J. Weis and M. L. Klein, Phys. Rev. Lett. 51, 670 (1983).

${ }^{37}$ D. Frenkel, Phys. Rev. Lett. 56, 858 (1986).

${ }^{38}$ N. Mikhin, A. Polev, E. Rudavskii and Y. Vekhov, J. Low Temp. Phys. 148, 707 (2007).

${ }^{39}$ V. N. Grigor'ev, N. P. Mikhin, E. Y. Rudavskii and Y. O. Vekhov, J. Low Temp. Phys. 150, 47 (2008).

${ }^{40}$ T. Markovich, E. Polturak, J. Bossy and E. Farhi, Phys. Rev. Lett. 88, 195301 (2002).

${ }^{41}$ A. Eyal, O. Pelleg, L. Embon and E. Polturak, Phys. Rev. Lett. 105, 025301 (2010).

${ }^{42}$ L. M. Sesé, J. Chem. Phys. 108, 9086 (1998).

${ }^{43}$ L. M. Sesé, J. Chem. Phys. 116, 8492 (2002); Mol. Phys. 101, 1455 (2003).

${ }^{44}$ L. M. Sesé and L. E. Bailey, J. Chem. Phys. 119, 10256 (2003).

${ }^{45}$ L. M. Sesé, J. Chem. Phys. 121, 3702 (2004).

${ }^{46}$ L. E. Bailey and L. M. Sesé, J. Chem. Phys. 121, 10076 (2004).

${ }^{47}$ L. M. Sesé, J. Chem. Phys.123, 104507 (2005).

${ }^{48}$ L. M. Sesé, J. Chem. Phys. 130, 074504 (2009). 
${ }^{49}$ L. M. Sesé, J. Chem. Phys. 136, 244504 (2012).

${ }^{50}$ H. F. Trotter, Proc. Am. Math.Soc. 10, 545 (1959); E. Nelson, J. Math. Phys. 5, 332 (1964).

${ }^{51}$ M. Fierz, Phys. Rev. 106, 412 (1957).

52 (a) D. Frenkel and B. Smidt, Understanding Molecular Simulation (Academic Press, San Diego, 2002); (b) C. Vega and E. G. Noya, J. Chem. Phys. 127, 154113 (2007); (c) J. L. Aragonés, C. Valeriani and C. Vega, J. Chem.. Phys. 137, 146101 (2012); d) M. Polson, E. Trizac, S. Pronk and D. Frenkel, J. Chem.. Phys. 112, 5339 (2000); e) R. Ramírez and C. P. Herrero, J. Chem.. Phys. 129, 064110 (2008).

${ }^{53}$ G. Navascués and E. Velasco, J. Chem. Phys. 132, 134106 (2010).

${ }^{54}$ L. M. Sesé, J. Phys. Chem. B 112, 10241 (2008).

${ }^{55}$ M. J. Mandell, J. P. McTague and A. Rahman, J. Chem. Phys. 66, 3070 (1977).

${ }^{56}$ R. Melrose and K. Singer, Mol. Phys. 66, 1203 (1989).

${ }^{57}$ P. J. Steinhardt, D. R. Nelson and M. Ronchetti, Phys. Rev. B 28, 784 (1983).

${ }^{58}$ See supplementary material at [URL will be inserted by AIP] for the complete numerical results.

${ }^{59}$ D. Scharf, G. J. Martyna and M. L. Klein, J. Chem. Phys. 99, 8997 (1993); G. J. Martyna, A. Hughes and M. E. Tuckerman, J. Chem. Phys. 110, 3275 (1999).

${ }^{60}$ J. Morales and K. Singer, Mol. Phys. 73, 873 (1991).

${ }^{61}$ M. E. Tuckerman, B. J. Berne, G. J. Martyna and M. L. Klein, J. Chem. Phys. 99, 2796 (1993);

M. E. Tuckerman, D. Marx, M. L. Klein and M. Parrinello, J. Chem. Phys. 104, 5579 (1996);.

${ }^{62}$ R. J. Speedy, J. Phys.: Condens. Matter 9, 8591 (1997).

${ }^{63}$ J. P. Hansen and I. R. McDonald, Theory of Simple Liquids (Academic, London, 1976).

${ }^{64}$ R. D. McCarty, J. Phys. Chem. Ref. Data 2, 923 (1973).

${ }^{65}$ L. Filion, M. Hermes, R. Ni and M. Dijkstra, J. Chem. Phys. 133, 244115 (2010).

${ }^{66}$ E. Kim and M. H. W. Chan, Nature (London) 427, 225 (2004). 
${ }^{67}$ R. Agrawal and D. A. Kofke, Mol. Phys. 85, 23 (1995); 85, 43 (1995).

${ }^{68}$ R. M. Lynden-Bell, Mol. Phys. 86, 1353 (1995).

${ }^{69}$ B. Smidt, K. Esselink and D. Frenkel, Mol. Phys. 87, 159 (1996).

${ }^{70}$ C. Chakravarty and R. M. Lynden-Bell, J. Chem. Phys. 113, 9239 (2000).

${ }^{71}$ T. Korona, H. L. Williams, R. Bukowski, B. Jeziorsky and K. Szalewicz, J. Chem.. Phys. 106, 5109 (1997).

${ }^{72}$ A. R. Janzen and R. A. Aziz, J. Chem. Phys. 107, 914 (1997).

${ }^{73}$ M. J. Cohen and J. N. Murrell, Chem. Phys. Lett. 260, 371 (1996). 
TABLE I. Reference structural data for the perfect $f c c, b c c$ and $h c p$ lattices. For computational convenience, the central boxes are assumed to be cubes in $f c c$ and $b c c$ and a rectangular parallelepiped in the $h c p$ case. Wave vectors are expressed as $\mathbf{k}=2 \pi\left(k_{x} / L_{x}, k_{y} / L_{y}, k_{z} / L_{z}\right)$, where $L_{x}, L_{y}$ and $L_{z}$ denote the three lengths of the box.

\begin{tabular}{|c|c|c|c|c|c|c|c|}
\hline Lattice & $Q_{4}$ & $Q_{6}$ & $N_{S}$ & & $k_{y}$ & $k_{z}$ & $S_{\max }(\mathbf{k})$ \\
\hline$f c c$ & 0.1909 & 0.5745 & $500 \quad(10 \times 10 \times 5)$ & $\begin{array}{c}-5 \\
5 \\
-5\end{array}$ & $\begin{array}{l}5 \\
5 \\
5\end{array}$ & $\begin{array}{r}5 \\
-5 \\
-5\end{array}$ & $\begin{array}{l}1 \\
1 \\
1\end{array}$ \\
\hline$b c c$ & 0.0364 & 0.5107 & $432 \quad(12 \times 6 \times 6)$ & $\begin{array}{l}6 \\
0 \\
6\end{array}$ & $\begin{array}{l}0 \\
6 \\
6\end{array}$ & $\begin{array}{l}6 \\
6 \\
0\end{array}$ & $\begin{array}{l}1 \\
1 \\
1\end{array}$ \\
\hline$h c p$ & 0.0972 & 0.4848 & $512 \quad(8 \times 8 \times 8)$ & $\begin{array}{l}0 \\
8 \\
4\end{array}$ & $\begin{array}{c}0 \\
0 \\
-8\end{array}$ & $\begin{array}{r}8 \\
-4 \\
4\end{array}$ & $\begin{array}{l}1 \\
0.75 \\
0.75\end{array}$ \\
\hline
\end{tabular}


TABLE II. Einstein crystal parameters utilized in this article. Hard-sphere collision parameter in this article $\sigma=3.5 \AA$.

\begin{tabular}{|c|c|c|c|c|c|}
\hline Lattice & $\rho_{N}^{*}$ & $\lambda_{B}^{*}$ & $N_{S} \times P$ & $\alpha_{M}\left(\AA^{-2}\right)$ & $c_{0}\left(\AA^{-2}\right)$ \\
\hline \multirow[t]{4}{*}{$f_{c c}$} & 0.8 & 0.2 & $500 \times 12$ & $1 \times 10^{5}$ & 2.058 \\
\hline & 0.7 & 0.4 & $500 \times 12$ & $7 \times 10^{4}$ & 2.130 \\
\hline & 0.6 & 0.6 & $500 \times 12$ & $1 \times 10^{4}$ & 1.502 \\
\hline & 0.6 & 0.8 & $500 \times 12$ & $1 \times 10^{4}$ & 2.153 \\
\hline \multirow[t]{4}{*}{$h c p$} & 0.8 & 0.2 & $512 \times 12$ & $1 \times 10^{5}$ & 1.992 \\
\hline & 0.7 & 0.4 & $512 \times 12$ & $7 \times 10^{4}$ & 2.105 \\
\hline & 0.6 & 0.6 & $512 \times 12$ & $1 \times 10^{4}$ & 1.488 \\
\hline & 0.6 & 0.8 & $512 \times 12$ & $1 \times 10^{4}$ & 2.136 \\
\hline \multirow[t]{6}{*}{$h c p$} & 0.7 & 0.4 & $64 \times 12$ & $3 \times 10^{4}$ & 2.376 \\
\hline & & & $216 \times 12$ & $3 \times 10^{4}$ & 2.203 \\
\hline & & & $512 \times 3$ & $3 \times 10^{3}$ & 2.035 \\
\hline & & & $512 \times 6$ & $7 \times 10^{3}$ & 2.098 \\
\hline & & & $512 \times 18$ & $7 \times 10^{4}$ & 2.075 \\
\hline & & & $512 \times 24$ & $1 \times 10^{5}$ & 2.098 \\
\hline
\end{tabular}


TABLE III. Einstein crystal results at $h c p\left(\rho_{N}^{*}=0.7 ; \lambda_{B}^{*}=0.4\right)$ for different sample sizes. $A=$ total Helmholtz free energy, $A_{i}=$ components of $A$ as defined in Eq. (8). Numbers in parentheses stand for one-standard deviation in the last decimal(s). 1 kpass $=10^{3} N_{S} \times P$ attempted bead moves.

\begin{tabular}{|l|l|l|l|l|l|l|l|}
\hline$N_{S} \times P$ & kpasses & $A / R T$ & $A_{1} / R T$ & $A_{2} / R T$ & $A_{3} / R T$ & $A_{4} / R T$ & $A_{5} / R T$ \\
\hline $64 \times 12$ & 6250 & $1.59(7)$ & 87.612 & $-85.964(74)$ & $0.2770(2)$ & -0.1723 & -0.1654 \\
\hline $216 \times 12$ & 1850 & $1.64(5)$ & 87.612 & $-86.140(53)$ & $0.2770(2)$ & -0.0511 & -0.0575 \\
\hline $512 \times 12$ & 800 & $1.66(6)$ & 102.864 & $-101.276(59)$ & $0.1187(1)$ & -0.0240 & -0.0268 \\
\hline $512 \times 3$ & 800 & $1.68(1)$ & 24.018 & $-22.342(12)$ & $0.0483(2)$ & -0.0189 & -0.0254 \\
\hline $512 \times 6$ & 800 & $1.67(2)$ & 43.185 & $-41.622(23)$ & $0.1484(1)$ & -0.0193 & -0.0261 \\
\hline $512 \times 12$ & 800 & $1.66(6)$ & 102.864 & $-101.276(59)$ & $0.1187(1)$ & -0.0240 & -0.0268 \\
\hline $512 \times 18$ & 800 & $1.65(6)$ & 132.400 & $-131.101(62)$ & $0.4008(4)$ & -0.0228 & -0.0272 \\
\hline $512 \times 24$ & 800 & $1.63(10)$ & 168.661 & $-167.649(96)$ & $0.6647(6)$ & -0.0230 & -0.0274 \\
\hline $512 \times 24$ & 5600 & $1.65(4)$ & 168.661 & $-167.630(39)$ & $0.6647(6)$ & -0.0230 & -0.0274 \\
\hline
\end{tabular}


TABLE IV. Results for the fluid-solid equilibria of quantum hard spheres $(\mathrm{F}=$ fluid, $\mathrm{S}=f c c, h c p)$. Numbers in parentheses are one-standard deviation in the last digit(s). Reduced pressures $p^{*}$ in units of $\left(\hbar^{2} / m \sigma^{5}\right)$. In this article: $m=28.0134$ amu, $\sigma=3.5 \AA$.

\begin{tabular}{|l|l|l|l|l|l|l|l|l|l|l|}
\hline$\lambda_{B}^{*}$ & System & $\rho_{F}^{*}$ & $\rho_{S}^{*}$ & $p^{*}$ & $G / R T$ & $E_{F} / R T$ & $E_{S} / R T$ & $S_{F} / R$ & $S_{S} / R$ & $\Delta \rho_{f}$ \\
\hline 0.2 & $\mathrm{~F}-f c c$ & $0.789(3)$ & $0.863(4)$ & $1566.49(2359)$ & $11.632(202)$ & $2.620(8)$ & $2.519(11)$ & $3.63(5)$ & $2.45(6)$ & 0.086 \\
\hline & $\mathrm{F}-h c p$ & $0.789(3)$ & $0.863(4)$ & $1563.64(2390)$ & $11.606(205)$ & $2.620(8)$ & $2.520(10)$ & $3.63(4)$ & $2.45(6)$ & 0.086 \\
\hline 0.4 & F $-f c c$ & $0.672(2)$ & $0.731(2)$ & $344.05(380)$ & $14.138(153)$ & $3.538(15)$ & $3.432(7)$ & $2.44(3)$ & $1.28(6)$ & 0.081 \\
\hline & F $-h c p$ & $0.671(3)$ & $0.730(3)$ & $342.23(420)$ & $14.070(166)$ & $3.529(15)$ & $3.425(11)$ & $2.45(3)$ & $1.29(6)$ & 0.081 \\
\hline 0.6 & F - $f c c$ & $0.589(3)$ & $0.635(3)$ & $140.05(196)$ & $16.118(198)$ & $4.381(16)$ & $4.309(12)$ & $1.88(4)$ & $0.83(6)$ & 0.072 \\
\hline & F - $h c p$ & $0.589(3)$ & $0.636(3)$ & $139.83(185)$ & $16.098(183)$ & $4.381(16)$ & $4.319(12)$ & $1.88(4)$ & $0.82(5)$ & 0.074 \\
\hline 0.8 & F - $f c c$ & $0.533(2)$ & $0.573(3)$ & $78.78(116)$ & $18.885(221)$ & $5.336(16)$ & $5.374(10)$ & $1.51(4)$ & $0.49(4)$ & 0.070 \\
\hline & F - $h c p$ & $0.534(3)$ & $0.574(3)$ & $79.37(153)$ & $19.002(285)$ & $5.357(16)$ & $5.391(12)$ & $1.50(4)$ & $0.48(5)$ & 0.070 \\
\hline
\end{tabular}


TABLE V. Representative integer components of maximizing commensurate centroid wave vectors for the QHS partially crystalline solids simulated with boxes that are cubes for $f c c$ and $b c c$-q and rectangular parallelepipeds for $h c p$. Hard-sphere parameter $\sigma=3.5 \AA$.

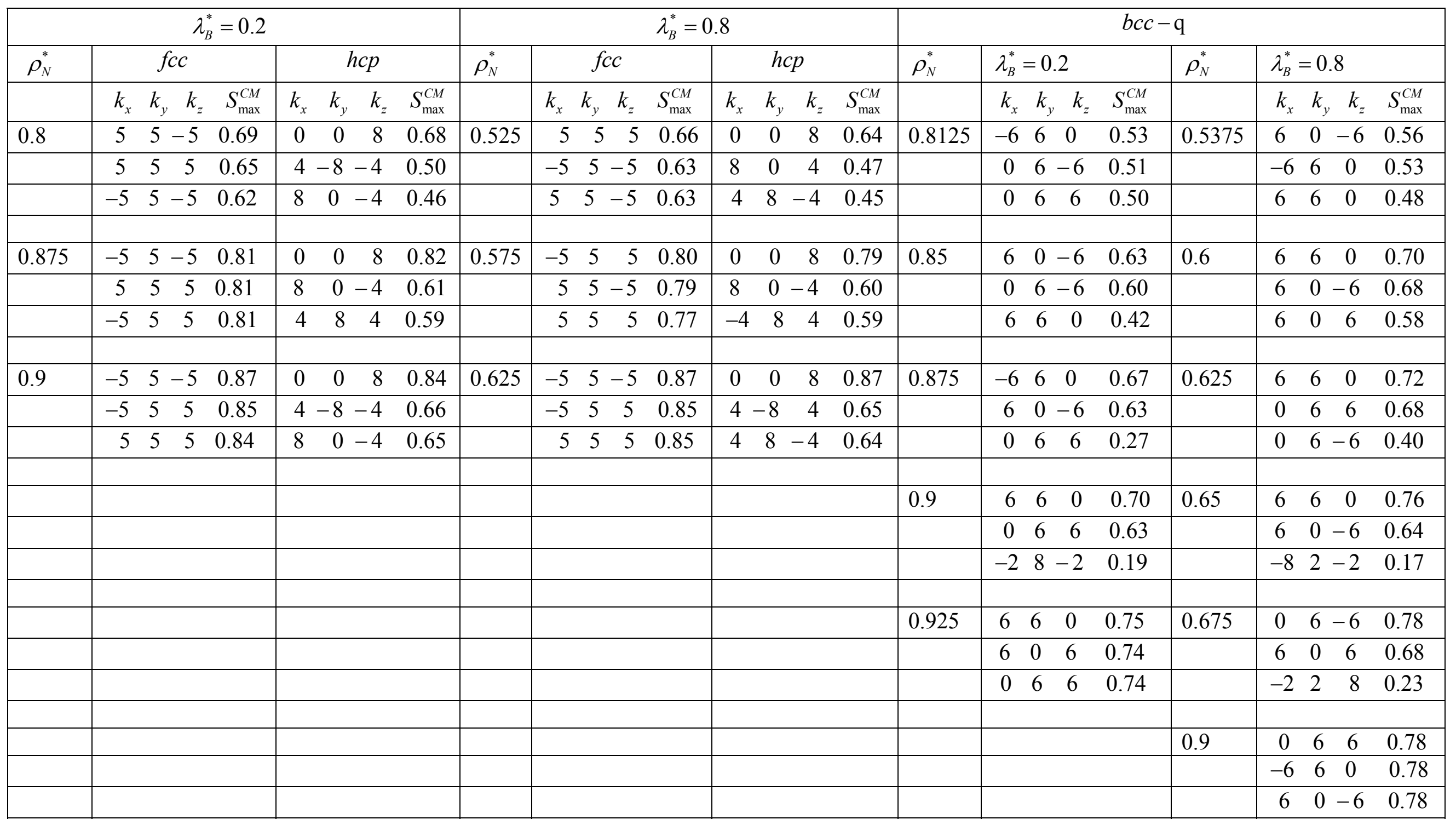




\section{Figure Captions}

\section{Figure 1.}

Typical pair radial correlation functions (GCM-2 = centroids; GET-2= instantaneouos) for $f c c$ partially crystalline state points. To complete the information the pair continuous linear response function GLR2 at one state point is also shown. The upper graphs are shifted by +6 . All the graphs are smoothed with B-splines. The dotted vertical lines mark the positions of the neighboring atoms in the corresponding $f c c$ perfect lattices (see main text). Their heights are a guide to the eye. Radial distance in reduced units $r^{*}=r / \sigma(\sigma=3.5 \AA)$.

\section{Figure 2}

The same as Fig. 1 but for hcp partially crystalline state points.

Figure 3

Reduced internal energies of the $f c c$ and $h c p$ partially crystalline state points investigated in this article. Also shown are the results for the fluid phase (Ref. 49).

\section{Figure 4}

The same as Fig. 3 but for the compression factors.

\section{Figure 5}

Coexistence fluid - solid $f c c$ curve for quantum hard spheres including data for $\lambda_{B}^{*}>0.8$ taken from Runge-Chester's work (Ref. 15). Also shown former 2007 results (Ref. 31) obtained via the calculation of isothermal compressibilities for the fluid and Eq. (2) -virial - for the solid. Note the logarithmic scale for reduced pressures $\left(p^{*}=p / \hbar^{2} m^{-1} \sigma^{-5}\right)$. The connecting lines are guides to the eye.

\section{Figure 6}

Comparison between the QHS current and 2007 (Ref. 31) data for reduced Gibbs free energies (black) and entropies (blue) for the range $\lambda_{B}^{*} \leq 0.8$. Error bars (one-standard deviation) also shown, and the smallness of the current ones is to be noted. In both cases the fluid-solid $f c c$ equilibrium are obtained via calculations of the fluid isothermal compressibilities. The connecting lines are guides to the eye. 


\section{Figure 7}

Comparison between the melting-freezing lines of QHS $(f c c-$ fluid $)$ and helium-4 for the reduced pressure (black) and the fluid number density (blue). Experimental data for ${ }^{4} \mathrm{He}$ taken from Ref. 64 , and $\sigma\left({ }^{4} \mathrm{He}\right)=2.2 \AA$ as defined in Ref. 15 . The connecting lines are guides to the eye.

Figure 8

Reduced internal energies (squares) and compression factors (circles) for the $b c c$-q state points investigated in this article. The dotted segments are a guide to the eye for identifying the non-regular behaviors along each isotherm.

Figure 9

The two typical forms of the pair radial correlation functions (GCM-2 and GET-2) for the bcc-qII and III state points. The upper plots have been obtained with 2760 kpasses and a sample size $432 \times 24$, and are shifted by +7 . All the graphs are smoothed with B-splines. The dotted vertical lines mark the positions of the neighboring atoms in the corresponding perfect $b c c$ lattice (see main text). Their heights are a guide to the eye. Radial distance in reduced units $r^{*}=r / \sigma \quad(\sigma=3.5 \AA)$.

Figure 10

Plots of the pair radial structures GCM-2 and GET-2 at $\left(\rho_{N}^{*}=0.8 ; \lambda_{B}^{*}=0.625\right)$ studied with $h c p$ and $b c c$-qII simulations. The upper plots are shifted by +5 . All the graphs are smoothed with B-splines. The dotted vertical lines mark the positions of the neighboring atoms in a perfect $h c p$ lattice at the same density (see main text). Their heights are a guide to the eye. Radial distance in reduced units $r^{*}=r / \sigma(\sigma=3.5 \AA)$.

Figure 11

Comparison between $b c c$-qII, $h c p$, and $f c c$ instantaneous pair radial correlation functions at the same conditions as those in Fig.10, where the $b c c \rightarrow b c c$-qII ( $h c p$-like) transition takes place. All the graphs are smoothed with B-splines. Radial distance in reduced units $r^{*}=r / \sigma \quad(\sigma=3.5 \AA)$. 
Figure 12.

Plots of the instantaneous structure of a typical $b c c$-qII state point at selected stages of its simulation by starting the calculations from the perfect $b c c$ lattice (the volume is kept constant). One $b c c-q$ block consists of 92 kpasses. The upper plots are shifted by +5 and +10 , respectively. The dash-dotted vertical lines mark the positions of the neighboring atoms in the corresponding perfect $b c c$ lattice (see main text). Their heights are a guide to the eye. Radial distance in reduced units $r^{*}=r / \sigma \quad(\sigma=3.5 \AA)$. 

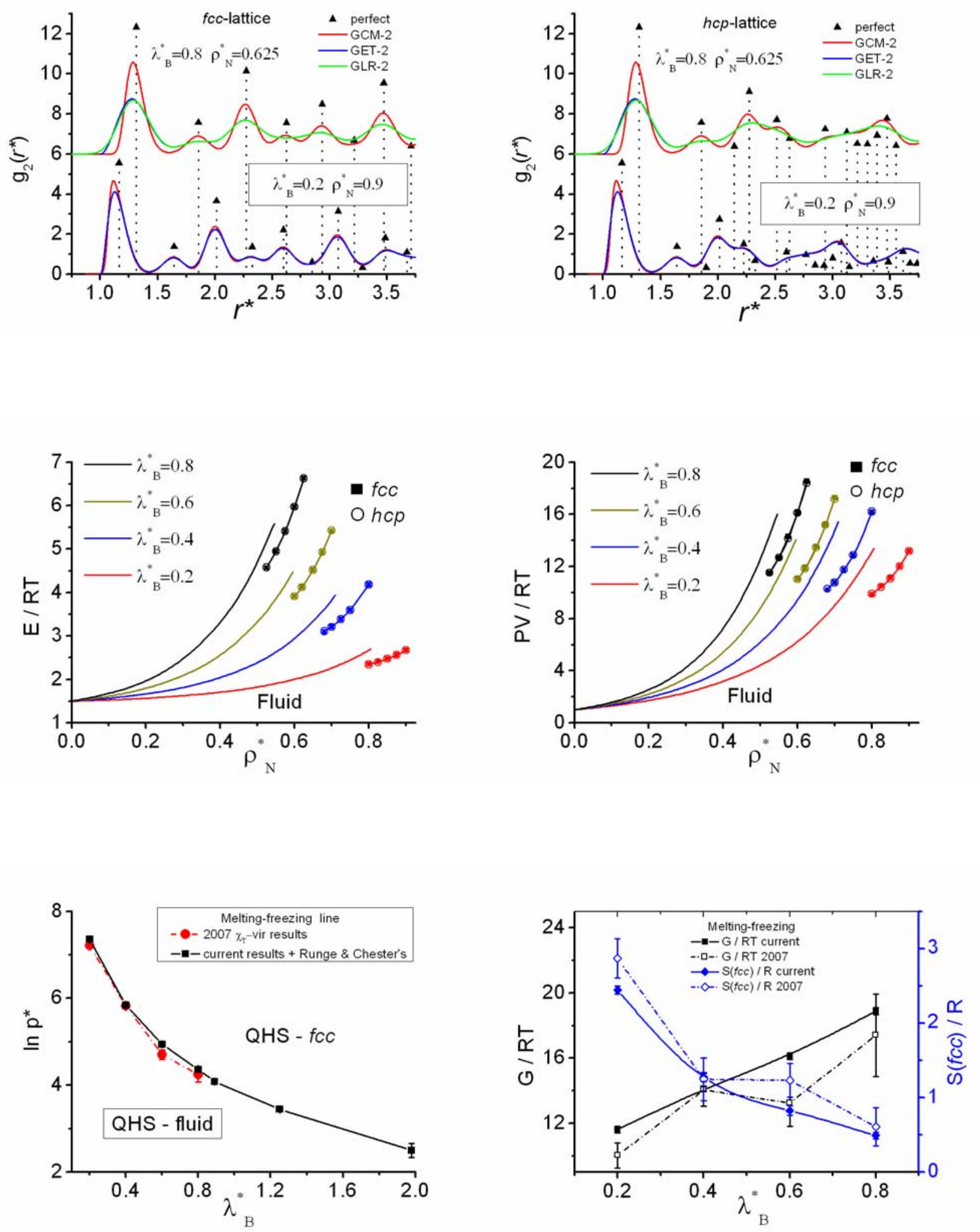

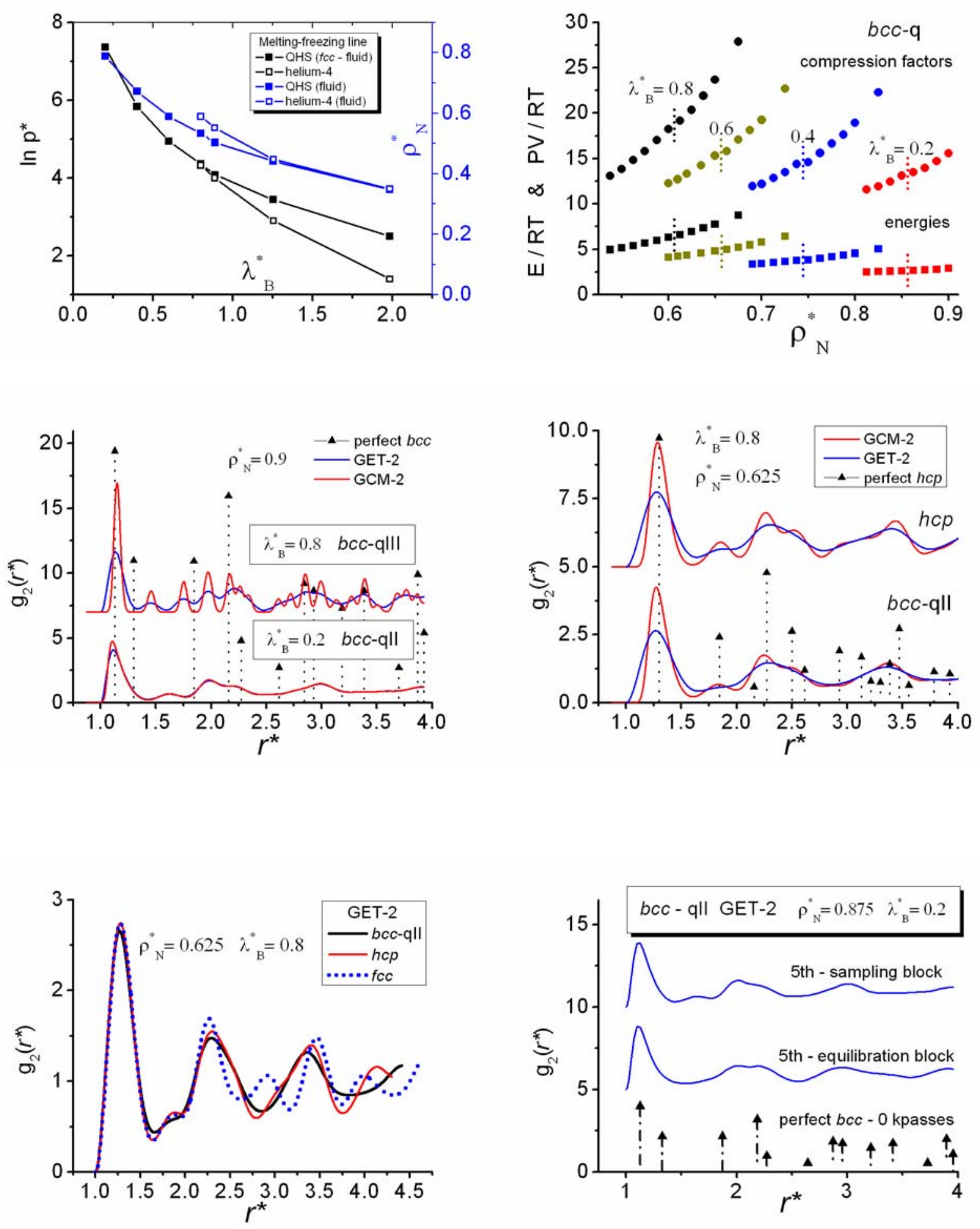